\section{Monitoring als Baustein für die Entscheidungsfindung in Endlagerprojekten}

\author{
Beate Kallenbach-Herbert und Stefan Alt, \\ Öko-Institut Darmstadt
}

Das Monitoring bei der Endlagerung erfolgt durch kontinuierliche oder wiederholte Messungen technischer und geologischer Parameter über längere Zeiträume. Die gewonnenen Daten dienen vielfältigen Zwecken. Ein zentrales Ziel besteht darin, Grundlagen für Entscheidungen zwischen den und innerhalb der verschiedenen Phasen eines Endlagerprojekts bereitzustellen. Monitoring ist somit nicht nur die Summe technischer Überwachungsmaßnahmen. Es tangiert auch wichtige Bereiche gesellschaftspolitischer Entscheidungen. Der Artikel beleuchtet mögliche Ziele von Monitoring, zeigt relevante technische Aspekte auf und zieht daraus Schlussfolgerungen zu Anforderungen an ein integriertes Monitoringkonzept. Es wird gezeigt, dass es notwendig ist, ein solches Monitoringkonzept frühzeitig und unter Einbeziehung aller an der Endlagerung beteiligten Akteure zu entwickeln.

\section{Einleitung}

Maßnahmen zur Überwachung sollen in allen Phasen eines Endlagerprojekts angewandt werden. Dies ergibt sich schon aus Gründen der bergrechtlichen und betrieblichen Sicherheit. Auch für den Strahlenschutz sind Messungen durchzuführen, sobald die ersten Abfälle eingelagert werden. Der Betreiber eines Endlagers kann in diesen Fällen auf weitgehend etablierte Methoden aus dem Bergbau und aus dem Arbeits- und Strahlenschutz zurückgreifen.

Ein deutlich komplexeres Bild entsteht, wenn man das Monitoring als Baustein der Entscheidungsfindung innerhalb eines Endlagerprojekts betrachtet. An wichtigen Meilensteinen eines Endlagerprojekts (z. B. wenn ein Standort endgültig festgelegt wird, wenn der Betrieb aufgenommen, der Verschluss begonnen oder die Überwachung eingestellt wird) sind Entscheidungsgrundlagen erforderlich, zu denen Monitoring-Daten beitragen. Wenn sich das Endlagerprojekt nicht wie erwartet entwickelt, können Betreiber oder Behörden mit Hilfe von Monitoring-Daten über erforderliche Interventionen entscheiden.

Derartige Entscheidungen werden zunehmend nicht allein auf der Basis naturwissenschaftlich-technischer Erkenntnisse gefällt. Sie erfordern vielmehr eine gesellschaftliche Verständigung, die politische Entscheider sowie Betroffene und Interessierte einbezieht. Um die mittels Monitoring gewonnenen Daten in diesen Entscheidungen sinnvoll nutzen zu können, ist es erforderlich, dass sich alle Beteiligten zuvor über Art, Umfang und Aussagekraft dieser Daten verständigt haben. Gleiches gilt für die „Erwartungswerte" oder Maßstäbe, die bei der Bewertung der gewonnen Daten angelegt werden. Monitoring ist also nicht nur ein technisches Konzept. Es tangiert auch wichtige Bereiche gesellschaftspolitischer Entscheidungen (s. Kuppler/Hocke in diesem Heft). Welche Anforderungen sich daraus für die Entwicklung eines Monitoringkonzepts ergeben, wird nachfolgend beleuchtet.

\section{Ziele des Monitorings}

Bisher wurden weder im nationalen noch im internationalen Bereich Empfehlungen entwickelt, wie ein Monitoring zu gestalten sei, das auch die gesellschaftspolitischen Anforderungen in den verschiedenen Phasen eines Endlagerverfahrens unterstützt. Empfehlungen der Internationalen Atomenergie-Organisation (IAEA) bleiben diesbezüglich allgemein und unverbindlich. Sie benennt zum einen den unspezifischen „Wunsch“ der Gesellschaft zur Teilhabe an Entscheidungsprozessen. Zum anderen werden der Verschluss des Endlagers und die Nachbetriebsphase als gesellschaftspolitisch relevante Schritte aufgeführt, in denen Monitoring-Daten potenziell eine Rolle spielen. In ihrem Safety Guide werden gesellschaftliche Implikationen im Wesentlichen für die Nachbetriebsphase erwähnt (IAEA 2011). Das Monitoring dient demnach in dieser Phase v. a. der Befriedigung öffentlichen Interesses und Sicherheitsbedürfnisses. Aus wissenschaftlich-technischer Sicht wird es für nicht erforderlich gehalten und daher als optional eingestuft. OECD-NEA befasst sich diesbezüglich ebenfalls 
nur mit den späten Phasen eines Endlagerprojekts (NEA 1999; NEA 2004).

In der aktuellen EURATOM-Richtlinie zur Entsorgung der radioaktiven Abfälle (EURATOM 2011) finden sich keine entsprechenden Hinweise. Die Sicherheitsanforderungen des Bundesumweltministeriums für die Endlagerung Wärme entwickelnder Abfälle (BMU 2010a) enthalten Vorgaben für ein Kontroll- und Beweissicherungsprogramm. Damit soll nachgewiesen werden, dass die Eingangsdaten und Annahmen der Sicherheitsanalysen und -nachweise eingehalten werden. Über die Integration gesellschaftspolitischer Anforderungen in dieses Programm finden sich keine Angaben.

In dem europäischen Forschungsvorhaben „MoDeRn“ wurde untersucht, welchen Stellwert das Thema Monitoring in den Diskussionsprozessen zur Endlagerung in den beteiligten Ländern hat. Demnach findet weder in Deutschland noch in den meisten anderen MoDeRn-Ländern (z. B. Großbritannien, Schweiz, Schweden) ein expliziter Austausch mit Vertretern aus Politik und Gesellschaft über die Erwartungen an ein Monitoring statt (MoDeRn 2011). Einzig in Großbritannien und der Schweiz hat das Forschungsteam erste Ansätze einer solchen Diskussion identifiziert. Zusammenfassend kommt der Bericht zu dem Schluss, dass weitere Schritte erforderlich seien, um belastbare Einschätzungen über die Erwartungen gesellschaftlicher Akteure an das Monitoring zu erzielen.

Nachfolgend werden daher zunächst Ziele diskutiert, die ein Monitoringkonzept, das einen sinnvollen Baustein bei der Entscheidungsfindung in den verschiedenen Phasen eines Endlagerprojekts darstellt, verfolgen sollte. Diese Ziele sind eng verbunden mit dem Endlagerkonzept und dem Prozess, der ein Endlagerprojekt leitet.

\subsection{Monitoring und Rückholbarkeit}

Ein einschlägiges Beispiel für den engen Zusammenhang zwischen Endlager- und Monitoringkonzept ist die Rückholbarkeit der Abfälle. Dieser wird auch im Summary Report des Projekts MoDeRn hervorgehoben: „A commitment to retrievability or reversibility may have significant implications for monitoring strategies."
(MoDeRn 2011, S. 13) Die Sicherheitsanforderungen des Bundesumweltministeriums legen fest, dass die Abfälle während der Betriebsphase rückholbar sein müssen (BMU 2010a). In den ersten 500 Jahren nach dem Verschluss soll es außerdem möglich sein, die Abfälle zu bergen, indem ein neues Bergwerk aufgefahren wird. ${ }^{1}$

Das Beispiel Asse zeigt, welch ein dynamischer Prozess aus politischen, gesellschaftlichen und wissenschaftlich-technischen Sichten sich bei der Entscheidung über die Rückholung entwickelt (s. z. B. Kallenbach-Herbert/Minans 2008). Sicher werden auch zukünftige Generationen eines Tages nicht rein technisch-wissenschaftlich darüber entscheiden, ob sie hochradioaktive Abfälle aus mehreren hundert Metern Tiefe zurückholen.

Die Option der Rückholbarkeit kann daher nur dann ein sinnvoller Baustein des Endlagerkonzepts sein, wenn Indikatoren vorhanden sind, auf deren Basis zukünftige Betreiber und (politische) Entscheider entscheiden können. Diese Indikatoren müssen sowohl die naturwissenschaftlich-technische als auch die gesellschaftlich-politische Dimension umfassen. Es ist daher wichtig, dass alle Interessengruppen sich darüber verständigen, welche Monitoring-Daten erforderlich sind, wie diese ermittelt werden können und wie mit den Ergebnissen weiter verfahren wird.

\subsection{Entscheidungsgrundlage im Endlagerverfahren}

Transparente Entscheidungen in einem Endlagerverfahren sind nur möglich, wenn die notwendigen Entscheidungsgrundlagen vorhanden sind. Diese umfassen einerseits die erforderlichen Daten, andererseits müssen sich die Beteiligten darüber verständigen, wie diese Daten interpretiert und an welchen Maßstäben sie gemessen werden.

Beispielsweise spielen bei der Standortauswahl tektonische Daten wie Hebungen und Senkungen oder hydraulische Gegebenheiten wie Grundwasservorkommen eine Rolle. Damit der Betreiber einen Standort bewerten kann und eine Behörde oder politische Entscheider die Standortauswahl treffen können, muss klar sein, wie und mit welcher Aussagekraft diese Daten erhoben und interpretiert werden. Außerdem muss festgelegt sein, welche Hebungen oder Senkungen zulässig 
sind. Ein transparentes, als legitim empfundenes Verfahren setzt voraus, dass diese Vereinbarungen getroffen werden, bevor die entsprechenden Erkundungsschritte beginnen. Für den „Arbeitskreis Auswahlverfahren Endlagerstandorte" (AkEnd) ist die frühzeitige Festlegung von Kriterien und Bewertungsmaßstäben (,Bewertungsgrundsätze") von umfassender Bedeutung für ein Standortauswahlverfahren (AkEnd 2002, S. 65ff.).

Die Diskussion in Deutschland um das Modell des „einschlusswirksamen Gebirgsbereichs“ (AkEnd 2002) verdeutlicht, dass bereits bei der Vereinbarung der zu erhebenden Daten die wissenschaftlichen, technischen, gesellschaftlichen und politischen Erfordernisse und Erwartungen einzubeziehen sind: Während einige Akteure den einschlusswirksamen Gebirgsbereich als maßgeblichen Faktor für den Einschluss der radioaktiven Stoffe annehmen, betonen andere die Notwendigkeit einer zweiten geologischen Barriere (s. z. B. BÜNDNIS 90/Die Grünen 2011). Entsprechend gehen auch die Einschätzungen auseinander, welche Kennwerte entscheidungserheblich sind und welche Daten ein Monitoring erfassen sollte.

\subsection{Bestätigung von Annahmen der Auslegung und Nachweisführung}

Während des gesamten Endlagerverfahrens - auch zwischen den Meilensteinen - dient das Monitoring dazu, zu prüfen, ob sich die Geologie und das Endlager erwartungsgemäß verhalten: Nur wenn beim Auffahren von Einlagerungsbereichen die geologische Situation den Prognosen entspricht, können die Abfälle planmäßig dort eingelagert werden. Nur wenn das vorgesehene Versatzmaterial, mit dem Hohlräume verfüllt werden sollen, die vorgesehene Dichtwirkung erzielt, wird es in den Einlagerungsbereichen verwendet werden. Für Betreiber und Behörde liefert das Monitoring somit die Grundlagen, um das geplante Vorgehen an die tatsächlichen Gegebenheiten anzupassen.

In ähnlicher Weise trägt das Monitoring auch zur Weiterentwicklung der Nachweisführung, insbesondere hinsichtlich der Langzeitsicherheit bei. Gemäß internationalen Empfehlungen (z. B. IAEA 2011) sollte ein standortspezifischer Langzeitsicherheitsnachweis (,post closure safety case") als Bestandteil des Safety Case erstmals im
Rahmen der Planung eines Endlagers erstellt und für jede nachfolgende Entscheidung aktualisiert werden: „The safety case should be prepared for at least each major step in the development, operation and closure of a geological disposal facility.“ (IAEA 2011, S. 18). Dabei wird von einer bereits vollzogenen Standortentscheidung ausgegangen.

Die Nachweisführung in einer frühen Projektphase muss zwangsläufig verschiedene Annahmen treffen, da die Erkenntnisse auf (untertägigen) Erkundungen in einem begrenzten Bereich der geologischen Formation beruhen. Wenn beispielsweise in der Phase der Errichtung und des Betriebs weitere Bereiche erschlossen werden, können zuvor getroffene Annahmen bestätigt oder widerlegt werden. Die aktuellen Sicherheitsanforderungen des Bundesumweltministeriums legen in Abschnitt 7.4 fest, welche Parameter in einem Kontroll- und Beweissicherungsprogramm mindestens zu erfassen sind (BMU 2010a).

\subsection{Weitere Ziele des Monitorings}

Da in Deutschland öffentliche Diskurse zum Monitoring bisher nicht stattgefunden haben, ist unklar, welche weiteren Ziele aus gesellschaftlicher Sicht damit verfolgt werden sollten. Auch die Hypothese, die beispielsweise im Bericht der IAEA aufgestellt wird (IAEA 2001), dass Monitoring das Vertrauen der Öffentlichkeit in die Langzeitsicherheit eines Endlagers stärken kann, kann daher für Deutschland weder bestätigt noch widerlegt werden.

Es ist zu erwarten, dass die regionale Bevölkerung insbesondere mit der Entscheidung zum endgültigen Verschluss eines Endlagers Erwartungen an die Überwachung von Schutzgütern verbindet. An erster Stelle steht dabei vermutlich die Überwachung des Grundwassers auf aus dem Endlager freigesetzte Radionuklide. Aber auch die Kontrolle von Geländeabsenkungen mit Auswirkungen auf Gebäude oder Kulturgüter könnte für Anwohner wichtig sein. Welche Erwartungen an das Monitoring als Grundlage für die Entscheidungsfindungen bestehen, wäre ebenso wie andere Monitoringziele in entsprechenden Diskussionsprozessen zu klären. Nachfolgend werden relevante Aspekte der technischen Realisierung von Monitoring zusammengefasst sowie 
derzeitige Möglichkeiten und Grenzen beim Monitoring aufgezeigt.

\section{Monitoring in den verschiedenen Phasen eines Endlagerprojekts}

Unter Monitoring werden hierbei kontinuierliche oder wiederholte Messungen über längere Zeiträume verstanden. Die Ausführungen zielen nicht darauf ab, mögliche Monitoringmaßnahmen umfassend zu analysieren. Vielmehr soll der Rahmen skizziert werden, in dem sich derzeitige Überlegungen zur Umsetzung von Monitoringmaßnahmen bewegen. Sie können als Hintergrundinformationen für einen interdisziplinären Diskurs zum Monitoring verstanden werden.

\subsection{Erkundung}

Die Erhebung von spezifischen Daten über konkrete Standorte beginnt mit der Erkundung von über Tage aus. Mittels Bohrungen und geophysikalischen Untersuchungen werden Informationen über die Gesteinseigenschaften und die geologische Situation erhoben. $\mathrm{Zu}$ den Informationen über die Gesteinsformationen gehören u. a. die hydraulische Durchlässigkeit sowie gebirgsmechanisches, thermisches und geochemisches Verhalten, zu denen über die geologische Situation Informationen zur Tiefe und Größe der für das Endlager vorgesehenen Gesteinsschicht sowie Störungen und Grundwasservorkommen in der Nähe zum potenziellen Endlagerbereich. Diese Daten werden in einem vergleichenden Verfahren herangezogen um zu entscheiden, welche Standorte vertieft zu erkunden und welche auszuschließen sind.

Bereits in dieser Phase sind auch erste Maßnahmen zum Monitoring des potenziellen Endlagerstandorts erforderlich. Viele Parameter, die an sich erst in einem fortgeschrittenen Stadium des Endlagerprojekts relevant sind, erfordern Messungen des jeweiligen Ausgangszustands, die später als Referenzdaten verwendet werden können. Hierzu zählen beispielsweise die Vermessung der Geländeoberfläche, die Temperaturverteilung im Untergrund oder der Spannungszustand des Gebirges. Wichtig sind auch Charakte- risierung und Überwachung des Grundwassers im Standortumfeld. Um mögliche Veränderungen des Grundwassers durch den Eintrag von chemischen Stoffen, Radionukliden oder durch Temperaturerhöhung genau erheben zu können, ist dessen ursprünglicher Zustand vor Beginn der untertägigen Erkundung zu erfassen. Das Monitoring dieses für alle Sicherheitsbetrachtungen zentralen Schutzgutes beginnt also frühzeitig und muss bis zu einem noch undefinierten Zeitpunkt nach Verschluss des Endlagers fortgeführt werden.

Mit fortschreitender Erkundung können die Monitoringmaßnahmen verfeinert und ausgebaut werden. So können z. B. erst im Zuge der untertägigen Erkundung konkrete Daten über potenzielle Einlagerungsbereiche erhoben werden. Hierzu werden Schächte, Strecken und Kammern aufgefahren und über einen längeren Zeitraum beobachtet (z. B. im Hinblick auf Standfestigkeit, Gebirgstemperatur, bergbaubedingte Auflockerung, Konvergenz und Reaktion auf die Bewetterung) oder als Ausgangsbasis für die Erkundung potenzieller Einlagerungsbereiche genutzt. Geophysikalische Methoden kommen zum Einsatz, um eine Interpretation der in den Erkundungshohlräumen gewonnenen Daten hinsichtlich ihrer räumlichen Ausdehnung zu ermöglichen.

\subsection{Errichtung und Betrieb}

Während der Errichtung und des Betriebs eines Endlagers sind die Arbeitsbedingungen hinsichtlich der konventionellen Arbeitssicherheit im Bergbau und des Strahlenschutzes beim Umgang mit den radioaktiven Abfällen zu überwachen. Außerdem ergeben sich Anforderungen an die radiologische Umgebungsüberwachung durch Messung der Radioaktivität, die mit der Abluft aus dem Schacht emittiert wird. Diese Anforderungen sind gut definiert (BMU 2010b) und in kerntechnischen Anlagen vielfach erprobt.

In der Betriebsphase des Endlagers ergibt sich eine Situation, in der Bereiche des Endlagers in unterschiedlichen Stadien nebeneinander bestehen. Für neu aufgefahrene Hohlräume ist die Konformität des Bereichs mit den Endlagereigenschaften nachzuweisen, wobei die Untersuchungen und Untersuchungsmethoden der untertägigen Erkundung fortgeschrieben 
werden. Mit der Einlagerung der radioaktiven Abfälle müssen weitere Anforderungen an die messtechnische Überwachung definiert werden, die sich aus dem Temperatureintrag durch die Abfälle sowie aus der Überwachung der Abfallgebinde im Hinblick auf die Einhaltung der Strahlenschutzgrenzwerte und die Integrität der Behälter ergeben. Nach der Versiegelung bereits gefüllter Einlagerungshohlräume und -bereiche tritt die Integritätsüberwachung von Versatz und technischen Barrieren hinzu, die zusammen mit der Überwachung von Druck (Versatzdruck, Gebirgsdruck, Spannungszustände, Porendruck), Gebirgsbewegungen (Konvergenz, Verschiebungen, Seismik), Temperatur und Feuchte Gegenstand des Monitorings sein wird. Ob weitere Parameter im Hinblick auf den Zustand der Abfallbehälter zur Beurteilung der Rückholbarkeit beobachtet werden müssen oder können, ist noch weitgehend offen. Kritikalitätssicherheit und Handhabbarkeit (Bergbarkeit) sind vor der Einlagerung nachzuweisen. Sie lassen sich nach der Einlagerung nicht mehr direkt beobachten.

\subsection{Versiegelung von Einlagerungshohlräumen}

Mit der Versiegelung von Einlagerungshohlräumen ergeben sich neue technische Anforderungen an das Monitoring. War es bisher möglich, installierte Messanordnungen dauerhaft instand zu halten, mit Energie zu versorgen und Daten zu gewinnen, so müssen Ermittlung und Übertragung von Daten aus einem verschlossenen Endlagerbereich nun unter der Prämisse erfolgen, dass die Integrität der geologischen und technischen Barrieren inkl. der Abfallbehälter nicht kompromittiert werden darf. Eine kabelgeführte Energie- und Signalübertragung oder Sensoren innerhalb von technischen Barrieren sind damit ausgeschlossen. Die installierte Technik kann nicht mehr gewartet oder ersetzt werden, es sei denn entsprechende Interventionsmaßnahmen wären erforderlich. Eine Zusammenfassung des Stands der Technik der unter diesen Bedingungen einsetzbaren Mess-, Datenübertragungs- und Energieversorgungssysteme wurde von Jobmann et. al. (2011) erstellt. Obwohl das technische Potenzial, Monitoring-Daten auch aus einem ver- schlossenen Endlager zu erhalten demzufolge vorhanden ist, setzen die technische Lebensdauer der eingesetzten Geräte und die Möglichkeiten einer autarken Energieversorgung dieser Art von Beobachtung zeitlich Grenzen. Eine direkte Überwachung der Verhältnisse in einem Endlager ist derzeit nur für Teilbereiche und über Zeiträume von einigen Jahrzehnten vorstellbar. Bei einem, mehrere Jahrzehnte dauernden Endlagerbetrieb ist absehbar, dass die Messsysteme in den ältesten Einlagerungsfeldern bereits bei der Stilllegung des Endlagers ausgefallen sind oder vor dem Ende ihrer technischen Lebensdauer stehen.

\section{$3.4 \quad$ Verschluss}

Mit der Beendigung der Abfalleinlagerung und der Versiegelung des letzten Einlagerungsbereichs steht der Verschluss des Endlagers an. Den Schachtverschlüssen kommt dabei eine besondere Bedeutung zu, da mit ihnen die einzigen technischen Durchdringungen der geologischen Barriere (und damit die einzigen Verbindungen zur Umwelt) langzeitsicher abgedichtet werden sollen. Eine Überwachung der Integrität der Schachtverschlüsse hinsichtlich gebirgsmechanisch relevanter Parameter ist technisch analog zum Monitoring der Einlagerungsbereiche zu realisieren.

\subsection{Nachbetriebsphase}

Nach dem Verschluss bleibt zunächst der Anspruch bestehen, das Endlagerverhalten zu überwachen, um die Konformität mit der erwarteten Entwicklung zu bewerten. Dies gilt insbesondere während des Zeitraums, in dem die Möglichkeit bestehen soll, die Abfallbehälter zu bergen (in Deutschland: 500 Jahre nach Verschluss). Da Informationen aus dem verschlossenen Endlager heraus voraussichtlich nur für einige Jahrzehnte verfügbar sein werden, werden indirekte Beobachtungen den Informationsbedarf decken müssen. Direkt auf das Endlagerverhalten zurückzuführen sind dabei Senkungen (konvergenzbedingt) oder Hebungen (temperaturbedingt) oberhalb des Endlagers (z. B. Tholen et al. 2008). Sinnvoller Weise werden die für die Überwachung benötigten Messpunkte an der Tagesoberfläche bereits vor Errichtung des 
Erkundungsbergwerks, also sehr früh im Endlagerprozess, festgelegt und für eine lange Überwachungsperiode gesichert bzw. instand gehalten. Messtechnisch können sie durch geodätische und geophysikalische Methoden überwacht werden (s. z. B. GRS 2006, S. 24), die auch satellitengestützt angewendet werden können.

Auch die Grundwasserqualität im Umfeld des Endlagers und in der Nähe der Schächte kann zur indirekten Beobachtung beitragen. Spätestens seit der übertägigen Erkundung sollten kontinuierliche Messreihen der vom Endlager unbeeinflussten Grundwasserqualität vorliegen, die dann mit späteren Überwachungsdaten verglichen werden können.

\section{Schritte zu einem integrierten Monitoringkonzept}

Das Monitoring, d. h. die wiederholte oder kontinuierliche Messung und Auswertung geologischer und technischer Daten an einem (potenziellen) Endlagerstandort, dient vielfältigen Zwecken: Bergbauliche und radiologische Parameter werden überwacht, um die betriebliche Sicherheit und den Strahlenschutz der Beschäftigten zu gewährleisten. Mit der Umgebungsüberwachung werden mögliche Auswirkungen eines Endlagers beispielsweise auf das Grundwasser und die Geländeoberfläche erfasst. Für zentrale Entscheidungen bei der Standortauswahl und bei Meilensteinen zwischen den verschiedenen Phasen eines Endlagerverfahrens werden Datengrundlagen bereitgestellt. Mit den zunehmenden Kenntnissen, die mit dem Monitoring gewonnen werden, werden zuvor getroffene Annahmen bestätigt oder widerlegt, so dass Analysen und Nachweise zunehmend fundiert werden können.

Es ist daher notwendig, ein Konzept zum Monitoring zu entwickeln, dass die verschiedenen Ziele und Ansprüche berücksichtigt. Dabei sind - v. a. im Hinblick auf die Relevanz für gesellschaftspolitisch relevante Entscheidungsprozesse - die folgenden Aspekte zu berücksichtigen.

- Bei der Entwicklung des Monitoringkonzepts, müssen die verschiedenen an der Endlagerung beteiligten Akteure einbezogen werden. So können einerseits die technischen Möglichkei- ten und Grenzen des Monitorings berücksichtigt werden und andererseits die absehbaren Erwartungen zukünftiger (politischer) Entscheider und gesellschaftlicher Gruppen von vornherein integriert werden. Entsprechende Diskussionsprozesse unterstützen das gegenseitige Verständnis und können dazu beitragen, dass das Monitoring einen wirksamen Beitrag für zukünftige Entscheidungen leisten kann.

- Bereits zu einem frühen Zeitpunkt, beginnend mit der übertägigen Erkundung potenzieller Endlagerstandorte, ist die Gewinnung von geologischen Daten und Umweltdaten von Bedeutung. Dies gilt sowohl für Erkenntnisse, die dazu dienen, Standorte zu bewerten und zu vergleichen, als auch für die Erfassung von Referenzparametern, die den unbeeinflussten Zustand beispielsweise des Grundwassers beschreiben. Ein Monitoringkonzept muss daher frühzeitig entwickelt werden, spätestens bevor die übertägigen Erkundungen im Rahmen eines Standortauswahlverfahrens beginnen.

- Wenn ein Endlagerkonzept mit Rückholbarkeit vorgesehen ist, muss das Monitoringkonzept so gestaltet sein, dass es Indikatoren bereitstellt, die die Entscheidung über eine ggf. erforderliche oder gewünschte Rückholung unterstützen können. Das Monitoringkonzept sollte also möglichst zeitnah zum Endlagerkonzept entwickelt werden.

- Neben den Parametern, die mit dem Monitoring erfasst werden sollen, sind auch Bewertungsmaßstäbe erforderlich, an denen die erhobenen Daten gespiegelt werden. Auch über diese ist eine breite Verständigung sinnvoll, da Betreiber oder Entscheider auf dieser Basis beispielsweise darüber entscheiden, ob der nächste Schritt des Prozesses aufgenommenen werden kann, ob Interventionen erforderlich sind oder ob Annahmen revidiert werden müssen.

Bisher wurde in Deutschland - ebenso wie in den meisten anderen Ländern, die die geologische Endlagerung planen - noch kein interdisziplinärer Diskurs über Erwartungen, Ziele oder Konzepte des Monitorings geführt. Die beschriebenen Zusammenhänge zeigen, dass ein solcher Diskurs, der möglichst alle relevanten Akteursgruppen einbezieht, hinsichtlich einer effektiven 
Nutzung von Monitoringdaten notwendig ist und frühzeitig erfolgen sollte.

Ein solcher Diskurs könnte beispielsweise im Zusammenhang mit der Diskussion über die „Entscheidungsgrundlagen“ für das Standortauswahlverfahren erfolgen (vgl. § 9 des aktuellen Entwurfs zum Standortauswahlgesetz; BMU 2012). Ein geeigneter Rahmen wäre beispielsweise eine interdisziplinäre Begleitgruppe mit dem Mandat, Hinweise zu Händen des Verfahrensführers zu erarbeiten. Dies könnte ergänzt werden durch Maßnahmen zum Austausch mit einem breiteren Kreis interessierter Akteursgruppen und der Öffentlichkeit.

\section{Anmerkung}

1) Auffahren meint, dass mit bergtechnischen Mitteln ein horizontaler oder geneigter Grubenraum geschaffen wird. (Anm. d. Red.)

\section{Literatur}

AkEnd - Arbeitskreis Auswahlverfahren Endlagerstandorte, 2002: Auswahlverfahren für Endlagerstandorte. Empfehlungen des AkEnd, Köln

BMU - Bundesministerium für Umwelt, Naturschutz und Reaktorsicherheit, 2010a: Sicherheitsanforderungen an die Endlagerung wärmeentwickelnder radioaktiver Abfälle. Bonn (Stand 30.9.10)

BMU - Bundesministerium für Umwelt, Naturschutz und Reaktorsicherheit, 2010b: Messanleitungen für die Überwachung der Aktivitätsableitungen radioaktiver Stoffe mit der Fortluft aus kerntechnischen Anlagen (Stand Juli 2010); http://www.bmu.de/strahlenschutz/ueberwachung_der_umweltradioaktivitaet/ messanleitungen/doc/41750.php (download 19.12.12) BMU - Bundesministerium für Umwelt, Naturschutz und Reaktorsicherheit, 2012: Gesetz zur Suche und Auswahl eines Standortes für ein Endlager für Wärme entwickelnde radioaktive Abfälle und zur Änderung anderer Gesetze. Bonn (Entwurf vom 17.10.2012)

BÜNDNIS 90/Die Grünen, 2011: Alternative Standortuntersuchung hätte Gorleben entwertet. PUABericht vom 25.3.2011 zur 39. Sitzung des 1. Parlamentarischen Untersuchungsausschusses Gorleben; http://www.gruene-bundestag.de/themen/gorlebenua/alternative-standortuntersuchung-haette-gorlebenentwertet_ID_376147.html (download 19.12.12)

EURATOM - Europäische Atomgemeinschaft, 2011: Richtlinie 2011/70/Euratom des Rates vom 19.7.2011 über einen Gemeinschaftsrahmen für die verantwortungsvolle und sichere Entsorgung abgebrannter Brennelemente und radioaktiver Abfälle; http://eurlex.europa.eu/LexUriServ/LexUriServ.do?uri=OJ:L :2011:199:0048:0056:DE:PDF (download 19.12.12)

GRS - Gesellschaft für Anlagen und Reaktorsicherheit $\mathrm{mbH}$, 2006: Gesamtbewertung der Langzeitsicherheit für den Standort Asse (Konsequenzenanalyse). GRS - A - 3350, Colenco-Bericht 3762/01

IAEA - International Atomic Energy Agency, 2001: Monitoring of Geological Repositories for High Level Radioactive Waste (IAEA-TECDOC-1208). Wien

IAEA - International Atomic Energy Agency, 2011: Geological Disposal Facilities for Radioactive Waste (Specific Safety Guide No. SSG-114). Wien

Jobmann, M.; Eilers, G.; Haverkamp, B. et al., 2011: Untersuchung geeigneter Methoden für das Monitoring eines Endlagers in tiefen geologischen Formationen von der Oberfläche aus und in der Betriebs- und frühen Nachbetriebsphase. Abschlussbericht. Peine: DBE Technology

Kallenbach-Herbert, B.; Minans, A., 2008: Unterstützung des BMU im Verfahren zur Stilllegung des Forschungsbergwerkes Asse II. Abschlussbericht (31.3.2008). Darmstadt: Öko-Institut

MoDeRn-Monitoring Developments for Safe Repository Operation and Staged Closure, 2011: National Monitoring Contexts - Summary Report; http://www. modern-fp7.eu/fileadmin/modern/docs/Reports/ MoDeRn_MonitoringContext_SummaryReport.pdf (download 10.12.12)

NEA - Nuclear Energy Agency, 1999: Confidence in the Long-term Safety of Deep Geological Repositories, its Development and Communication. Paris

NEA - Nuclear Energy Agency, 2004: Post-Closure Safety Case for Geological Repositories. Paris

Tholen, M.; Hippler, J.; Kreienmeyer, M. et al., 2008: Überprüfung und Bewertung des Instrumentariums für eine sicherheitliche Bewertung von Endlagern für HAW, ISIBEL Arbeitspaket 7, Gemeinsamer Bericht von DBE Technology, BGR und GRS. Peine

\section{Kontakt}

Dipl.-Ing. Beate Kallenbach-Herbert

Öko-Institut e.V.

Rheinstraße 95, 64295 Darmstadt

Tel.: +49 (0) 6151 / 819 - 119

E-Mail: b.kallenbach@oeko.de 